\begin{tabular}{|c|c|c|c|c|c|}
\hline Revista Praxis & ISSN: $1657-4915$ & Vol. 14 & No. 1 & $41-50$ & enero - junio de 2018 \\
\hline \multicolumn{6}{|c|}{ DOI: http://dx.doi.org/10.21676/23897856.2539 } \\
\hline
\end{tabular}

\title{
DIFICULTADES EN PROCESOS LECTO-ESCRITORES DE NIÑOS CON NET EN SEGUNDO GRADO DE PRIMARIA Y SU INCIDENCIA EN EL DESEMPEÑO ACADÉMICO-SOCIAL
}

\author{
LITERACY DIFFICULTIES OF SECOND GRADERS WITH TRANSITORY EDUCATIVE \\ NEEDS TEN AND THEIR INFLUENCY IN THEIR ACADEMICAL AND SOCIAL \\ PERFORMANCE
}

\author{
Gloria Stella Malagón-Pinzón ${ }^{(\mathbb{D})}$, Diego Orlando Aponte-Chirivii ${ }^{(\mathbb{D})}$, Astrid Viviana Rodríguez-Sierra ${ }^{3}$
}

\begin{abstract}
RESUMEN
Este artículo es el resultado de una investigación realizada con el propósito de analizar cómo influyen las dificultades lectoescritoras en el desempeño académico e interacción social de niños con Necesidades Educativas Transitorias (NET) que cursan segundo de primaria. Este estudio se desarrolló bajo una metodología de investigación mixta, aplicando en su fase cuantitativa una prueba diseñada por el Instituto para la Investigación Educativa y el Desarrollo Pedagógico (IDEP), y en su fase cualitativa, la observación con un nivel de baja estructuración. Los resultados de esta investigación permitieron detectar las dificultades de los estudiantes en la comprensión y la expresión oral, en el código escrito y en la interacción con pares y adultos. Además, se evidenció que estas inciden en el desempeño de las otras áreas del conocimiento y, por consiguiente, en los resultados académicos. El estudio brinda herramientas para detectar, dar respuesta e intervenir a través de estrategias pedagógicas los casos de estudiantes que no han alcanzado un desarrollo del lenguaje de acuerdo con su edad y el nivel que cursan.
\end{abstract}

Palabras clave: procesos lecto-escritores; necesidades educativas especiales; necesidades educativas transitorias; interacción social; desempeño académico.

\section{ABSTRACT}

This article is the result of a research project carried out to analyze the way literacy difficulties of children with Transitory Educative Needs (TEN) influence their academical performance and social interaction. A mixed methodology was followed by applying a test designed by Instituto para la Investigación y el Desarrollo (IDEP) in its quantitative phase, and the observation with a low structuration level in its qualitative phase. This research results allowed to detect students' difficulties in relation to their oral comprehension and expression, as well as in the written code and in the interaction with pairs and adults. Furthermore, it was evidenced that these difficulties affect the performance in other areas of knowledge and thus in the academic results. The study provides tools to detect, to give answers and to intervene through pedagogical strategies in the cases of students that have not reached the language development level according to their age and course.

Keywords: Literacy Processes; Special Educative Needs; Transitory Educative Needs; Social Interaction; Academic Performance.

Fecha de recepción: junio 21 de 2017 / Fecha de aceptación: junio 19 de 2018 / Publicado en línea: junio 20 de 2018 Tipología: Artículo de Investigación Científica y Tecnológica

Para citar éste artículo: Malagón, G., Aponte, D. y Rodríguez, A. (2018). Dificultades en procesos lecto-escritores de niños con NET de segundo grado de primaria y su incidencia en el desempeño académico-social. Praxis, 14(1), 41-50.

Doi: http://dx.doi.org/10.21676/23897856.2539

${ }^{1}$ Mg. Corporación Universitaria Minuto de Dios - UNIMINUTO, Bogotá, Colombia. Correo electrónico: glorismapi@gmail.com 0RCID: 0000-0002-4396-7882 ${ }^{2}$ Mg. Corporación Universitaria Minuto de Dios - UNIMINUTO,, Bogotá, Colombia. Correo electrónico: daponte2@uniminuto.edu 0RCID: 0000-0002-8397-5748 ${ }^{3}$ Ph.D. Cambiar por: Corporación Universitaria Minuto de Dios - UNIMINUTO, Bogotá, Colombia. Correo electrónico: avrodriguez@uniminuto.edu 0RCID: 0000-0001-7079-1168 


\section{INTRODUCCIÓN}

$E^{1}$ lenguaje como medio que permite interactuar con los demás y conocer el mundo, en ocasiones no alcanza un desarrollo adecuado de acuerdo con la edad de los estudiantes, quienes llegan a la escuela con una serie de dificultades que requieren respuesta e intervención. Estas Necesidades Educativas Especiales, en adelante NEE, se presentan cuando el estudiante en su interacción no alcanza el nivel del grupo porque su ritmo de aprendizaje es distinto al de sus compañeros de clase.

Cuando se utiliza el término NEE, es importante aclarar que incluye a los estudiantes que requieren más atención que otros en la misma clase, y que estas pueden ser temporales o permanentes. Una vez sean detectadas de acuerdo con las características y el contexto de los estudiantes, deben conectarse con la "respuesta educativa, que persigue el máximo desarrollo personal, intelectual, social y emocional del alumnado, de acuerdo con los objetivos establecidos con carácter general” (Luque, 2009, p.213).

Las NEE pueden ser de dos clases: transitorias, cuando se presentan en un período de escolaridad; o permanentes, cuando acompañan a la persona durante toda su vida. En su informe investigativo, Pinto, Olivera y Fuentes (2008) consideran dentro de las NEE Permanentes la discapacidad intelectual, auditiva, visual, motora, multidéficit, psíquica, graves alteraciones en la capacidad de relación y comunicación, y visceral. Mientras que forman parte de las Necesidades Educativas Transitorias (NET) el trastorno emocional, las fobias, la violencia intrafamiliar, el embarazo adolescente, la drogadicción, los trastornos específicos del aprendizaje, el aprendizaje lento, el déficit atencional, la hiperactividad, los trastornos conductuales, la deprivación socio-económica y cultural y los trastornos específicos del lenguaje.

En este sentido, la presente investigación se centra en las NET asociadas al lenguaje, espe- cíficamente en los procesos lecto-escritores relacionados con la producción oral y escrita de oraciones o textos sencillos, partiendo del vocabulario, de las estructuras gramaticales y de los procesos relacionados con la comprensión del lenguaje que permiten entender palabras, oraciones o textos de acuerdo con las destrezas y habilidades lectoras de los estudiantes de segundo de primaria de la Institución Educativa Distrital (I.E.D.) en Cundinamarca.

Estas dificultades que presentan los estudiantes han incidido en su desempeño académico en las diferentes áreas del conocimiento y en el intercambio comunicativo con su entorno. En la educación primaria dichas dificultades "representan un considerable porcentaje dentro de las dificultades de aprendizaje en general [...] El alumno con dificultades en lectoescritura no solo tiene problemas en el área de Lengua y Literatura, sino también en el resto de las áreas" (Celdrán y Zamorano, 2011, p.1).

Desde esta perspectiva, surge la siguiente pregunta: ¿cómo influyen las dificultades lectoescritoras que presentan los niños con NET de grado segundo de primaria en su desempeño académico e interacción social? Esta pregunta plantea la importancia de identificar de manera temprana las dificultades que presentan los estudiantes, determinando e implementando estrategias pedagógicas que ayuden a superar estas dificultades y mejoren el desempeño académico en general y los procesos de comunicación de los estudiantes.

La educación exige grandes retos. Uno de estos es reconocer que dentro del aula de clase pueden encontrarse estudiantes con diferentes necesidades educativas, quienes tienen las mismas oportunidades que los demás y requieren una intervención pedagógica enfocada en mejorar sus condiciones de aprendizaje y su calidad de vida. Por lo tanto, la educación como un derecho y una acción continua en la formación, presente en todas las etapas de la vida, debe brindar acceso a toda población sin excepción alguna. 


\section{Procesos lecto-escritores}

En cuanto al desarrollo del lenguaje, muchas son las necesidades educativas que impiden construir a los estudiantes su competencia comunicativa, lo que incide en su desempeño a la hora de escuchar, hablar, conversar, leer y escribir. Por esta razón, se debe tomar en cuenta que la comunicación ocurre tanto en medios orales como escritos, de modo que es fundamental el fortalecimiento de la producción comunicativa como de la interpretación comunicativa.

Además, en los procesos lecto-escritores, la escuela debe prestar atención a las necesidades, ritmos y estilos de aprendizaje de los estudiantes, es decir, se debe atender a la diversidad en los procesos de aprendizaje que cada estudiante tiene. Como lo plantea León (2007), los procesos de lectura y escritura deben enmarcarse dentro de la diversidad y reconocer las características de cada individuo, su desarrollo integral y antecedentes lingüísticos.

Leer y escribir son actividades complejas que forman parte de un mismo proceso y constituyen herramientas para la comunicación y el aprendizaje. "Al leer y al escribir (y al hablar y al escuchar) los niños y las niñas aprenden también a usar el lenguaje en su calidad (y en su cualidad) de herramienta de comunicación entre las personas y entre las culturas" (Lomas, 2013, p.7).

Escribir implica que el niño sea competente comunicativamente, es decir, que posea un desarrollo de la motricidad fina, una madurez intelectual para codificar y decodificar mensajes, un desarrollo del lenguaje de acuerdo con la edad y un dominio de las propiedades del texto como la coherencia, la cohesión, las formas gramaticales, la presentación para comprender lo que escribe y trasferir de significados.

Leer es una actividad cognoscitiva que implica interpretar textos según diferentes propósitos comunicativos, haciendo uso de los conocimientos previos para establecer una interacción entre esos significados y los propios. Es un proceso complejo que requiere enfrentar a los niños desde el comienzo de su vida escolar a una gran variedad de textos gráficos, escritos o mixtos, que puedan relacionar con sus vivencias, emociones, conocimientos para construir saberes que gradualmente los lleven a una lectura crítica de los textos que encuentran en los medios escritos y los que ofrecen las nuevas tecnologías.

El niño construye su lenguaje a partir del diálogo, el consenso, la colaboración, la lectura y la escritura en todas las situaciones que vivencia en su mundo familiar, escolar y social, es decir que el significado se construye a partir de la interacción social.

\section{METODOLOGÍA}

Este trabajo de investigación se desarrolló bajo una metodología mixta, en tanto utilizó elementos cualitativos y cuantitativos que responden al planteamiento del problema y a los objetivos propuestos. Otra de las razones por las cuales se consideró adecuada esta metodología, es su fácil adaptabilidad al diseño secuencial explicativo, porque inicialmente se recolectaron y analizaron los datos cuantitativos y luego los datos cualitativos, cruzando la información obtenida en instrumentos, como registros de observación dentro y fuera del aula, notas de campo, lista de chequeo, rejilla de valoración y prueba del proyecto "Valoración y abordaje de procesos de desarrollo, aprendizaje y sus dificultades", diseñada en el año 2015 por el Instituto para la Investigación Educativa y el Desarrollo Pedagógico (IDEP) con el fin de analizar los procesos de aprendizaje y las dificultades del ciclo inicial, debido a la incidencia de este período educativo en el aprendizaje y la educación de niños y niñas, de las instituciones públicas de Bogotá, Distrito Capital (Blanco et al., 2015).

El método de investigación cuantitativa facilitó la recolección de información acerca de las dificultades que presentan los estudiantes. A través su aplicación, se realizó el conteo y la medición numérica para observar la cantidad de niños con hallazgos en sus procesos lecto-escritores, se depuró la información, se tabularon los datos, se interpretaron 
los resultados con el apoyo del marco teórico y, por último, se propusieron estrategias pedagógicas conducentes a fortalecer dichos procesos y la interacción en el aula.

La observación como metodología cualitativa, con un nivel de baja estructuración, permitió obtener información acerca de la interacción con pares y con docentes, la participación en clase y las conversaciones que sostienen los estudiantes. Se hizo uso de esta metodología porque los anteriores aspectos no se pueden reducir fácilmente a variables ya que estos se evidencian y manifiestan de acuerdo con la situación comunicativa en la que los estudiantes se encuentren. La observación se realizó con una lista de chequeo y tomando notas de campo en diferentes momentos de la jornada escolar, haciendo registros y comentarios de las características más relevantes del comportamiento de cada estudiante. Los indicadores que se incluyeron en la lista de chequeo son:

- Conversa de manera espontánea con pares y adultos, manifestando sus necesidades, intereses, emociones y manteniendo el contacto visual.

- Utiliza algunas formas socialmente establecidas para iniciar y finalizar una conversación para saludar, despedirse, agradecer, solicitar un favor, etc.

- Escucha las intervenciones de sus compañeros y docentes, usando gestos que evidencian su atención, levanta la mano para pedir la palabra y espera su turno para intervenir.

- Responde preguntas con claridad y de forma completa.

- Formula preguntas cuando no comprende un mensaje o solicita ayuda a un compañero o docente.

- Expresa un propósito comunicativo en situaciones reales o imaginarias, de forma verbal y gestual

- Narra eventos de manera secuencial, usando palabras que indican orden en el tiempo (primero, luego, enseguida, después, finalmente).

- Sigue las instrucciones dadas en pocos pasos y trabaja en grupos pequeños manteniendo relaciones de colaboración.

\section{Participantes}

Los participantes o unidades de análisis del estudio de la presente investigación fueron 30 estudiantes, divididos en 14 niñas y 16 niños con NET, pertenecientes a las cuatro aulas de grado segundo de primaria, matriculados en la I.E.D. Colegio Cundinamarca cuyas edades oscilan entre los 7 y 8 años de edad.

El muestreo se hizo de manera intencional, la población objeto de este estudio se identificó con el apoyo del servicio de Orientación y los criterios que se tuvieron en cuenta para la selección de la muestra fueron los siguientes:

- Los estudiantes han cursado los grados cero, primero y segundo que forman el ciclo 1 en la institución, lo que permite un mayor conocimiento de sus dificultades y da validez a este estudio.

- Los resultados académicos de esta población en sus procesos lecto-escritores durante los años de escolaridad antes mencionados y su incidencia en el desempeño académico en general.

- La evaluación de necesidades académicas y de convivencia, que se hace al iniciar cada año escolar, para observar si los estudiantes han avanzado o si persisten sus dificultades.

\section{RESULTADOS}

La información se organizó por categorías, a partir de la prueba de lecto-escritura y la transcripción de las observaciones no estructuradas, realizadas a los estudiantes. Estas categorías son: comprensión y expresión oral, el código escrito y la interacción entre pares y con adultos.

\section{Comprensión y expresión oral}

En esta categoría se reúnen 3 indicadores: articulación, fluidez verbal, coherencia y comprensión de instrucciones. La tabla 1 ilustra los da- 
tos consolidados acerca de las dificultades que presentan los estudiantes con NET al articular algunos fonemas, los cuales se evidencian en su lectura y escritos. Una de las dificultades más grandes es la expresión oral con diferentes in- tenciones comunicativas y la adaptación a su interlocutor cuando entablan una conversación. Es importante aclarar que es un proceso en el cual el estudiante poco a poco con ayuda en el aula o de su terapeuta puede superar estas dificultades.

Tabla 1. Resultados obtenidos en la categoría: Comprensión y expresión oral.

\begin{tabular}{|l|c|c|c|c|c|c|}
\hline \multirow{2}{*}{ Indicador } & \multicolumn{5}{|c|}{ Valoración } \\
\cline { 2 - 7 } & E & $\%$ & B/A & \% & EP & $\%$ \\
\hline Articulación & 10 & 33,3 & 11 & 36,7 & 9 & 30 \\
\hline Fluidez verbal & 6 & 20 & 14 & 46,7 & 10 & 33,3 \\
\hline Coherencia & 10 & 33,3 & 15 & 50 & 5 & 16,7 \\
\hline Comprensión de instrucciones & 2 & 6,7 & 12 & 40 & 16 & 53,3 \\
\hline
\end{tabular}

Las equivalencias en la escala de valoración son: E: Excelente, B/A: Bueno con apoyo, EP: En proceso. *Se presenta el número de estudiantes y el porcentaje correspondiente en cada indicador de acuerdo con la escala de valoración. Fuente: elaboración propia basada en rejilla de evaluación del IDEP (2015)

\section{El código escrito}

La tabla 2 permite observar los datos consolidados con respecto a esta categoría e integra los siguientes indicadores: representación gráfica, relación entre grupos de palabras, completar frases, escritura espontánea, estructura gramatical y lectura de textos escritos. En esta categoría se evidenciaron aún más las dificultades que presentan los estudiantes para plasmar por escrito lo que leen: la letra es ilegible en algunos casos, en otros omiten letras, confunden, pero lo fundamental no se debe reducir a la forma, pues muchas de estas dificultades el estudiante las puede superar y corregir con el tiempo, a través del acercamiento al texto y a la necesidad que tiene como emisor para que los demás entiendan lo que quiere comunicar, lo que lo llevará a interiorizar las estructuras gramaticales, las normas ortográficas y la manera como conecta las palabras y las frases.

También se evidenció que los estudiantes con dificultades para comprender textos e instrucciones, se mostraron reticentes para escribir. Se observó temor e inseguridad a equivocarse en el momento de escribir espontáneamente, completar frases, relacionar grupos de palabras u organizar frases completas. Otros se preocuparon por la forma de la letra, la precisión, el manejo del renglón y no pudieron escribir frases completas, con concordancia de elementos o algunos conectores que indicaran tiempo.

Aunque los estudiantes se mostraron motivados al leer el texto, un porcentaje muy alto, el $70 \%$ de los mismos presenta déficits en sus procesos léxicos, porque se centran en la lectura de palabras y se les dificulta extraer el significado global del texto. 
Tabla 2. Resultados obtenidos en la categoría: Código escrito.

\begin{tabular}{|l|c|c|c|c|c|c|}
\hline \multirow{2}{*}{\multicolumn{1}{|c|}{ Indicador }} & \multicolumn{6}{c|}{ Valoración } \\
\cline { 2 - 7 } & $\mathbf{E}$ & $\mathbf{\%}$ & $\mathbf{B} / \mathbf{P}$ & $\mathbf{\%}$ & $\mathbf{E P}$ & $\mathbf{\%}$ \\
\hline Representación gráfica & 2 & 6,7 & 16 & 53,3 & 12 & 40 \\
\hline Relación entre grupo de palabras & 0 & 0 & 19 & 63,3 & 11 & 36,6 \\
\hline Completar frases & 3 & 10 & 12 & 40 & 15 & 50 \\
\hline Escritura espontánea & 2 & 6,7 & 18 & 60 & 10 & 33,3 \\
\hline Estructura gramatical & 1 & 3,3 & 19 & 63,3 & 10 & 33,3 \\
\hline Lectura & 0 & 0 & 9 & 30 & 21 & 70 \\
\hline
\end{tabular}

Fuente: elaboración propia basada en rejilla de evaluación del IDEP (2015)

\section{Interacción con pares y adultos}

Esta categoría enriqueció la investigación porque las observaciones realizadas en las actividades espontáneas de los estudiantes con pares y adultos, en este caso los docentes, ayudó a analizar el desarrollo de su lenguaje en la interacción con otros, ratificando los planteamientos de Vygotsky. En la escuela los estudiantes tienen muchas oportunidades de interactuar socialmente y estas interacciones son fundamentales para el desarrollo de sus capacidades lingüísticas. El significado del lenguaje se construye en la interacción social, ya sea por escrito u oralmente.

Vygotsky basó sus ideas sobre el lenguaje interior y del papel que éste juega en el dominio del comportamiento y en la conciencia, tanto en el análisis literario como en el análisis de la actividad concreta de los niños y de su comunicación con otros (Alonso, 2000, p.62).

Los estudiantes de grado segundo con NET disfrutan las actividades grupales, pero aún se les dificulta asumir algunos roles, seguir instrucciones, debido a los bajos niveles de escucha que hay entre ellos.
De acuerdo con los resultados obtenidos en la categoría de comprensión y expresión oral, se puede inferir que los estudiantes a pesar de las dificultades para articular algunos fonemas, realizar frases o textos cortos que tengan concordancia en tiempos o categorías que se relacionan como artículo-sujeto-verbo, se les facilita más la expresión oral que la escrita. Cuando se realizó la actividad oral, hubo mayor participación y motivación por parte de los estudiantes. Una de las dificultades que presentan es la comprensión de una serie de instrucciones, pues muchos realizan la actividad, mientras que otros las dejan sin terminar o simplemente no lo intentan.

En cuanto al manejo del código escrito, se encuentran textos en los que hay letras invertidas, poco manejo del espacio, unión de letras, fragmentaciones, adición, omisión y sustitución de letras, sílabas y palabras, pero al leer el texto este expresa el pensamiento del estudiante aunque no haya un claro propósito comunicativo, por lo tanto, no es prioridad la forma sino el 
mensaje que se quiere expresar, como lo explica Aguirre (2000):

(...) al afirmar que un niño no sabe escribir, casi siempre se habla de la apariencia de lo escrito, esto es, no usa letra con buena forma y bonita; en otras ocasiones se habla de la escritura como copia incorrecta, pero en muy contadas ocasiones, se piensa en su contenido o mensaje escrito (p.148).

El análisis nos lleva a pensar que la gramática quizá sea el eje central de los procesos lectoescritores y se dejen de desarrollar procesos como la comprensión y producción textual, una aproximación poco funcional como la plantea Ochoa (2008): “(...) la gramática aparece como una herramienta para apoyar los procesos de producción y comprensión de textos y no como un saber a desarrollar en sí mismo. El no trabajarla de esta manera impide el desarrollo de la competencia comunicativa” (p.12).

Otro aspecto fundamental que se evidencia es la falta de hábitos en la lectura, lo que trae como consecuencia un vocabulario limitado, falta de fluidez en la conversación, repetición de vocablos, falta de comprensión literal, inferencial y argumentativa, ya que los estudiantes deben leer muchas veces un texto para su análisis.

Finalmente, para los estudiantes, la palabra se debe convertir en la mediadora que les ayude a construir el sentido de lo que perciben y viven, y a la vez, les sirva para comunicarse e interactuar con los otros. Dentro de las observaciones se evidencia que para ellos es más fácil expresar sus emociones y sentimientos a través del lenguaje corporal y gestual que de forma verbal. De acuerdo con Acosta (2006):

Vygotsky mencionó el lenguaje como una forma de mediación fundamental para el desarrollo del conocimiento científico y cotidiano, ya que aunque tanto conceptos cotidianos como los científicos se desarrollan en la comunicación, unos fuera o dentro del ambiente educativo, el discurso en las instituciones educativas representa una forma de comunicación cualitativamente diferente, ya que las palabras actúan no sólo como medio de comunicación, como en el discurso cotidiano, sino como objeto de estudio (p.126).
En la interacción se logran aprendizajes colaborativos a partir de la ayuda de adultos o de pares, que permiten la zona de desarrollo próximo y la mediación en la acción humana de elementos como el lenguaje que modifican las estructuras cognoscitivas. Este proceso mental que llevan a cabo los individuos es producto de la interiorización de los aprendizajes culturales y sociales "entre los cuales ocupan un lugar central los signos o símbolos, tales como el lenguaje, los símbolos matemáticos, los signos de la escritura y, en general, todos los tipos de señales que tienen algún significado definido socialmente" (Briones, 2006, p.151).

Autores como Monereo y Solé (como se citó en Grañeras y Parras, 2008) sostienen que el enfoque educacional constructivo es el más adecuado para orientar la intervención psicopedagógica, porque la interacción permite que las personas adultas o más avanzadas medien entre el aprendiz y su entorno, para integrar y construir nuevos conocimientos, a partir de saberes previos, con actividades planificadas, significativas, promotoras de la zona de desarrollo potencial y ajustadas a las necesidades de los estudiantes.

\section{Estrategias pedagógicas}

Dentro y fuera del aula se deben favorecer los procesos de intercambio comunicativo, de hechos y situaciones del entorno próximo de los estudiantes, que enriquezcan su vocabulario, que sean coherentes, que atiendan a distintos propósitos comunicativos y a aspectos gramaticales de la Lengua Castellana (concordancia, tiempos, etc.). Por esta razón, se deben abrir espacios que involucren a los estudiantes con dificultades para expresarse oralmente, a través de actividades como el canto, la declamación, la dramatización, la narración de secuencias temporales, la lectura, los trabalenguas, los refranes, las retahílas, entre otros.

Otra estrategia consiste en corregir las expresiones verbales erróneas, ingenuas o incompletas de los estudiantes, sin recalcar en la equivocación, sino a través de la modelación. Si, 
por ejemplo, el estudiante ante la dificultad para articular un sonido lo cambia o invierte por otro ("cocholate": chocolate) y emite el siguiente enunciado: "El cocholate es una bebida deliciosa"; el docente puede retomar el ejemplo diciendo: "Por supuesto, el chocolate es una bebida deliciosa que nos da energía”. De esta manera, se modela la expresión aportando elementos gramaticales y semánticos. Adicionalmente, el docente puede plantear preguntas abiertas que progresivamente lleven al estudiante a ampliar su vocabulario o usar el parafraseo para saber si los estudiantes escuchan y comprenden lo que los compañeros o los docentes dicen.

También es importante promover la escritura espontánea de textos, para que los estudiantes plasmen sus vivencias, anécdotas, situaciones en familia y en su entorno con el uso del cuaderno viajero, como herramienta que ayuda a los estudiantes con dificultades para expresarse verbalmente, porque les permite evocar recuerdos y recuperar información de situaciones personales, familiares y escolares para luego escribirlas e ilustrarlas con fotos, dibujos, logos, etc. Otro recurso es el portafolio o carpeta personal que contiene todas las producciones escritas de los estudiantes: cuentos, fábulas, anécdotas, descripciones, historietas, recetas y sus actividades de comprensión lectora. El portafolio no es un archivo de trabajos, pues este debe permitir la reflexión y la retroalimentación. A través de la construcción de varios borradores sobre un escrito, el estudiante va puliendo y mejorando su redacción, ortografía y cohesión textual. Y por último, el escrituario, que promueve la escritura espontánea en un tiempo indicado sobre temas de interés como: películas, viajes comidas, juguetes, mascotas, deportes favoritos, seres que más aman, proyectos cuando sean grandes, el mejor amigo, los sueños, etc. Al finalizar la escritura de cada texto, los estudiantes cuentan el número de palabras escritas. Este ejercicio no se califica y si se realiza regularmente, los estudiantes amplían su vocabulario y mejoran su expresión escrita. El docente para generar confianza también puede escribir al tiempo con los estudian- tes y leer su escrito, esto animará a los que deseen compartir sus escritos.

Además, la lectura se debe hacer desde todas las áreas del conocimiento y no sólo desde el área de español y literatura, pues diferentes textos pueden leer e interpretar los estudiantes, ya sean narrativos, descriptivos, comparativos, instructivos, en fin, y como estrategia organizar la información de estos en esquemas o diagramas sencillos que les ayuden a comprender lo que leen.

Una buena estrategia es motivar e involucrar a los padres de familia en el proceso lecto-escritor de sus hijos, para que fomenten hábitos de lectura y escritura, asistan a las actividades en las que sus hijos se expresan oralmente o presentan sus producciones escritas.

\section{CONCLUSIONES}

Los instrumentos aplicados a los estudiantes de segundo de primaria con NET permitieron identificar las dificultades en los procesos de lectura y escritura que inciden directamente en su desempeño académico, ya que afectan la comprensión, interpretación y producción de contenidos propios de otras áreas del conocimiento y, por lo tanto, demandan respuesta e intervención por parte de docentes, tutores u orientadores a través del desarrollo e implementación de estrategias pedagógicas, con el fin de ayudar a los estudiantes a superar sus dificultades y a evitar que estas necesidades se vuelvan permanentes a lo largo de su formación académica y de su vida.

En las observaciones realizadas, a los estudiantes se les facilita más la expresión oral que la escrita, por esta razón, dentro de las estrategias se recomienda la apertura de espacios que promuevan el desarrollo de la expresión oral y la lectura de textos que respondan a diferentes propósitos comunicativos que sean significativos, acordes a sus necesidades e intereses, antes que tomar como punto de partida la escritu- 
ra, ya que a través de la lectura los estudiantes pueden ir asimilando las reglas sintácticas, los elementos semánticos, la correcta escritura de las palabras, la construcción de frases y de párrafos, el uso de los signos de puntuación y los matices afectivos de la voz, lo cual hará más fácil el camino de la escritura y evitará procesos mecánicos como las planas o la memorización de una serie de reglas gramaticales.

En el Colegio Cundinamarca I.E.D. la detección de las dificultades en los procesos lecto-escritores se hace a partir del diagnóstico de necesidades que se realiza al comenzar un curso, pero la intervención e implementación de las estrategias la mayoría de las veces se vuelve labor exclusiva del docente del área de Español, lo cual se debe replantear, para que desde todas las áreas del conocimiento, de manera interdisciplinar y transversal se desarrollen las habilidades comunicativas, reconociendo los saberes previos, incorporando el uso de situaciones del entorno y expresiones que así sean erróneas o ingenuas se pueden convertir en oportunidades de aprendizaje, para desarrollar el lenguaje oral y escrito y la interacción de los estudiantes.

Además, la institución acoge estudiantes con necesidades educativas en sus aulas regulares, como un proceso de inclusión y con el propósito de que se adapten a la dinámica escolar con otros compañeros que no presentan estas dificultades y a la vez se generen oportunidades, se respeten las diferencias individuales. Sin embargo, aún falta enfatizar en la flexibilización y adaptación del currículo acorde con los ritmos de aprendizaje de los estudiantes. Este proceso cuenta con el apoyo de una sola orientadora para el ciclo que reúne los grados: preescolar, primero y segundo, lo que dificulta el seguimiento de los casos, debido al gran número de estudiantes con NET.

En la investigación de campo se determinó que existen factores que influyen en el aprendizaje de la escritura, como: el desarrollo psicomotriz en general, la motricidad fina, la coordinación viso motora y la firmeza en el trazo, en tanto en la lectura se encuentran la discriminación visual y auditiva, el manejo del espacio y la estructura temporal. Aunque estos factores no son objeto de esta investigación, son de gran importancia y pueden ser usados para futuras investigaciones que respondan a los siguientes interrogantes: ¿cómo influyen estos factores en la adquisición de la escritura o el acercamiento a la lectura?, cuando estos factores no se tienen en cuenta en la primera infancia, ihacen más difícil el proceso lecto-escritor? o cuando se detecta a un estudiante con necesidades educativas transitorias, ¿es posible que el origen de sus dificultades se encuentre en un inadecuado manejo de estos factores?

Por otro lado, la implementación de estrategias pedagógicas no es solamente responsabilidad de los maestros y la orientadora de la institución, también los padres se deben involucrar en estos procesos, pues no se observó acompañamiento por parte de la familia en las actividades extracurriculares y los planes de mejoramiento acordados con los maestros. Los padres de familia generalmente se muestran confundidos, agobiados, se niegan a creer la situación o no saben qué hacer, optando en la mayoría de las veces por dejar esa tarea solo a la institución, lo cual acrecienta aún más el problema. Surge aquí un cuestionamiento con respecto al compromiso en la formación de sus hijos que tienen los padres: ¿mediante qué estrategias la institución puede involucrar en el proceso de formación a los padres de los estudiantes con NET?

Finalmente, este estudio pone a disposición de los docentes algunas estrategias pedagógicas y didácticas que se pueden poner en práctica en el aula de clase para superar las dificultades en los procesos lecto-escritores, pero es un estudio abierto que puede enriquecerse, modificarse o adaptarse de acuerdo con las necesidades y los ritmos de aprendizaje de los estudiantes de grado segundo, quienes en la institución cierran el ciclo 1 de enseñanza y requieren contar con unos procesos específicos para iniciar el ciclo 2 . 


\section{REFERENCIAS BIBLIOGRÁFICAS}

Acosta, M. (2006). El aprendizaje visto como un proceso de interacción social. La perspectiva Vygotskiana vista desde la complejidad. Revista Ciencias de la Educación, 1(27), 123-134. Recuperado de http://servicio.bc.uc. edu.ve/educacion/revista/volIn27/27-8.pdf

Alonso, L. (2000). El papel del lenguaje interior en la regulación del comportamiento, EDUCERE, 3(9), 61-68. Recuperado de www. saber.ula.ve/bitstream/123456789/19512/1/ articulo9-4-9.pdf

Aguirre, R. (2000). Dificultades de aprendizaje de la lectura y la escritura EDUCERE, 4(11), 147-150. Recuperado de www.redalyc.org/ articulo.oa? id $=35601102$

Blanco, Y., Zea, L., Ortíz, G., León, J., Albarracín, L., Amaya, G.,...Acuña, L. (2015). Desarrollo y aprendizaje en el ciclo inicial: Valoración y abordaje pedagógico Una reflexión a partir de la experiencia. Bogotá, Colombia: Instituto para la Investigación Educativa y el Desarrollo Pedagógico (IDEP), Cooperativa Editorial Magisterio.

Briones, G. (2006). Teorías de las ciencias sociales y de la educación: Epistemología. México D F.: Ed. Trillas.

Celdrán, M. y Zamorano, F. (2011). Dificultades en la adquisición de la lecto-escritura y otros aprendizajes. Recuperado de http://diversidad.murciaeduca.es/orientamur/gestion/ documentos/unidad24.pdf
Grañeras, M. y Parras, A. (Coords.). (2008). Orientación educativa: fundamentos teóricos, modelos institucionales y nuevas perspectivas. España: CIDE.

León, S. (2007). Abordaje de los procesos de lectura y escritura en una escuela: propuesta pedagógica en y para la diversidad. Revista Electrónica Educare, 1, 65-88. Recuperado de http://www.redalyc.org/articulo.oa?id $=194119235005$

Lomas, C. (2013). Aprender a comunicar(se) en las aulas. Recuperado de: http://rabida. uhu.es/dspace/bitstream/handle/10272/3508/ b15760844.pdf?..

Luque, D. (2009). Las necesidades educativas especiales como necesidades básicas. Una reflexión sobre la inclusión educativa. Revista Latinoamericana de Estudios Educativos, 39(3-4), 202-223. Recuperado de http://www. redalyc.org/articulo.oa?id $=27015078009$

Ochoa, L. (2008). Leer y escribir correctamente: La gramática y su relación con la lectura y la escritura. Revista del Instituto para la Investigación Educativa y el Desarrollo Pedagógico (IDEP), (15), 9-20.

Pinto, A., Olivera, E. y Fuentes, R. (2008). Modelo de gestión curricular de la escuela para el logro de aprendizajes significativos de estudiantes con necesidades educativas especiales transitorias basado en articulaciones efectivas desde el Lenguaje. (Informe final, Proyecto FONIDE No. 000231). Maule, Chile. 